PROCEEDINGS OF THE

AMERICAN MATHEMATICAL SOCIETY

Volume 130, Number 9, Pages 2515-2524

S 0002-9939(02)06488-2

Article electronically published on April 22, 2002

\title{
GENERAL BLOW-UPS OF THE PROJECTIVE PLANE
}

\author{
TOMASZ SZEMBERG AND HALSZKA TUTAJ-GASIŃSKA
}

(Communicated by Michael Stillman)

\begin{abstract}
We study linear series on a projective plane blown up in a bunch of general points. Such series arise from plane curves of fixed degree with assigned fat base points. We give conditions (expressed as inequalities involving the number of points and the degree of the plane curves) on these series to be base point free, i.e. to define a morphism to a projective space. We also provide conditions for the morphism to be a higher order embedding. In the discussion of the optimality of obtained results we relate them to the Nagata Conjecture expressed in the language of Seshadri constants and we give a lower bound on these invariants.
\end{abstract}

\section{INTRODUCTION}

Rational surfaces obtained by blowing up $\mathbb{P}^{2}$ in several points have been studied in algebraic geometry for a long time. Let $\pi: X \longrightarrow \mathbb{P}^{2}$ be the blowing up of $\mathbb{P}^{2}$ in $r$ points with exceptional divisors $E_{1}, \ldots, E_{r}$ and let $H=\pi^{*} \mathcal{O}_{\mathbb{P}^{2}}(1)$. Line bundles of the form

$$
L=d H-\sum_{i=1}^{r} k_{i} E_{i}
$$

have been addressed by many authors with respect to different properties. Here we are interested in properties of the morphism $\phi_{L}$ of $X$ to a projective space, defined by the linear system $|L|$, provided that such a morphism exists (i.e. that $|L|$ is base point free). Even in the simplest case $k_{1}=\cdots=k_{r}=1$ an optimal criterion for $|L|$ to define a morphism was obtained only recently by Coppens [8] section 3.3]. It states that $L$ is globally generated if $X$ is obtained from $\mathbb{P}^{2}$ by blowing up at most $r \leq h^{0}\left(\mathcal{O}_{\mathbb{P}^{2}}(d)\right)-3$ general points and $d \geq 7$.

On the other hand, again under the assumption that all $k_{i}$ are equal to 1 , Küchle [16] and independently $\mathrm{Xu} 22$ showed that $L$ is ample provided $L^{2}>0$ and $d \geq 3$. Recently Biran [6, Corollary 2.1.B] obtained a similar optimal result if all $k_{i}$ are equal to 2. In this direction we prove the following (not optimal) result valid for all $k$.

Theorem 3. Let $\pi: X \longrightarrow \mathbb{P}^{2}$ be the blowing up of $\mathbb{P}^{2}$ in $r$ general points and let $k \geq 2$ and $d$ be integers such that $d \geq 3 k+1$. If $r \leq \frac{d^{2}}{k^{2}}-1$, then the line bundle $L=d H-k \sum_{i=1}^{r} E_{i}$ is ample.

Received by the editors October 13, 2000 and, in revised form, March 30, 2001.

2000 Mathematics Subject Classification. Primary 14E25; Secondary 14C20.

The first author was partially supported by KBN grant 2 P03A 00816.

The second author was partially supported by KBN grant 2 P03A 01418. 
With ampleness of $L$ established it is natural to ask when $L$ is very ample. This problem also has a long history, e.g. [4], [5], the ultimate result (again in the case all $k_{i}=1$ ) being proved by D'Almeida and Hirschowitz [9. They showed that $\phi_{L}$ is an embedding if $X$ is obtained from $\mathbb{P}^{2}$ by blowing up at most $r \leq h^{0}\left(\mathcal{O}_{\mathbb{P}^{2}}(d)\right)-6$ general points and $d \geq 5$.

The notions of the global generation of a line bundle and the very ampleness were generalized in several ways in the past decade. Here we focus on the $k$-very ampleness (see Definition 1.1) which in a sense governs the geometry of secant varieties of $X$. Since a $k$-very ample line bundle has degree at least $k$ on any curve, a necessary condition for $L$ to be $k$-very ample is that $k_{i} \geq k$ for all $i=1, \ldots, r$ (for a slightly different approach see [20). Thus fat points necessarily come into the picture. If $r \leq 8$, i.e. if $X$ is a Del Pezzo surface, Di Rocco 11 obtained the complete characterization of $k$-very ample line bundles. It seems that for the greater number of points $r \geq 9$ no satisfactory generalizations of results of Coppens have been found. In this direction Theorem 4 provides a criterion for the global generation of $L$. The problem we study here was investigated by Ballico and Coppens 1, however their cohomological conditions seem difficult to verify in general. In this paper we restrict to the homogeneous case $k_{1}=\cdots=k_{r}=k$ and prove the following.

Theorem 1. Let $\pi: X \longrightarrow \mathbb{P}^{2}$ be the blowing up of $\mathbb{P}^{2}$ in $r$ general points and let $k$ and $d$ be non-negative integers such that $d \geq 4 k+1$. If $r \leq \frac{(d+3)^{2}}{(k+1)^{2}}-4$, then the line bundle $L=d H-k \sum_{i=1}^{r} E_{i}$ is $k$-very ample.

Our approach is a combination of methods used by Di Rocco [11 and those introduced by Ein and Lazarsfeld 12 and used by $\mathrm{Xu}[22$. This result does not seem to be optimal. After the proof we state a conjectural bound and discuss its relation to the famous Nagata Conjecture. Towards the Nagata Conjecture we prove the following bound.

Theorem 2. Let $P_{1}, \ldots, P_{r}$ be $r \geq 9$ general points in $\mathbb{P}^{2}$ and let $k_{1}, \ldots, k_{r}$ be fixed non-negative integers. If $C \subset \mathbb{P}^{2}$ is a curve of degree $d$ such that mult $_{P_{i}} C \geq k_{i}$, then

$$
d \geq \frac{1}{\sqrt{r+1}} \sum_{i=1}^{r} k_{i}
$$

This bound improves the previous result of Xu 21] (see Proposition 2.2) except in the case when $r+1$ is a square where the strong inequality follows from [22]. In roughly half of the cases the above bound also improves those following from results obtained by Harbourne [13].

Notation. We work throughout over the field $\mathbb{C}$ of complex numbers. If $X$ is a variety, by $K_{X}$ we denote the canonical divisor of $X$, and by $H^{i}(X, \mathcal{F})=H^{i}(\mathcal{F})$ the cohomology groups of a coherent sheaf $\mathcal{F}$ on $X$. The numerical equivalence of divisors is denoted by $\equiv$. For a given real number $\alpha$ we denote by $\lceil\alpha\rceil$ its roundup, i.e. the least integer greater than or equal to $\alpha$. By a very general point of an algebraic variety we mean a point lying away from a countable sum of Zariski closed subsets. 


\section{1. $K$-VERY AMPLENESS}

Recently there has been considerable interest in generalizations of the notions of the global generation and the very ampleness of a line bundle. For the purpose of the present note we recall the following.

Definition 1.1. Let $k$ be a non-negative integer. A line bundle $L$ on a projective variety $X$ is said to be $k$-very ample if the evaluation mapping

$$
H^{0}(L) \longrightarrow H^{0}\left(L \otimes \mathcal{O}_{Z}\right)
$$

is surjective for every 0 -dimensional subscheme $Z \subset X$ of length at most $k+1$.

Thus a line bundle is globally generated if and only if it is 0-very ample and it is very ample if and only if it is 1-very ample. Geometrically, if $X$ is embedded by a $k$-very ample line bundle, then there are no $(k+1)$-secant $(k-1)$-planes in the embedding. Equivalently, one obtains an embedding of the Hilbert scheme $S^{[k+1]} X$ into an appropriate Grassmanian; see [7] for details.

It follows at once from the definition that a $k$-very ample line bundle is $l$-very ample for every $l \leq k$. It is also easy to see that the tensor product $L_{1} \otimes L_{2}$ of a $k_{1}$-very ample line bundle $L_{1}$ with a $k_{2}$-very ample line bundle $L_{2}$ is $\left(k_{1}+k_{2}\right)$-very ample. Thus a naive way to obtain a result along the lines of Theorem 1 would be to use the result of D'Almeida-Hirschowitz mentioned in the Introduction. This might be formulated as follows.

Proposition 1.2 (A naive criterion). Let $\pi: X \longrightarrow \mathbb{P}^{2}$ be the blowing up of $\mathbb{P}^{2}$ in $r$ general points and let $k$ be a positive integer. If $r \leq \frac{(\lfloor d / k\rfloor+2)(\lfloor d / k\rfloor+1)}{2}-6$ and $d \geq 5 k$, then the line bundle $L=d H-k \sum_{i=1}^{r} E_{i}$ is $k$-very ample.

Roughly speaking this naive result is weaker by a factor $\frac{1}{2}$ than the result proved in Theorem 3,

On surfaces there is the following useful criterion of Beltrametti and Sommese [3] which generalizes Reider's theorem to $k$-very ample adjoint line bundles.

Proposition 1.3 (Beltrametti-Sommese). Let $X$ be a smooth surface and let $N$ be a nef line bundle on $X$, such that $N^{2} \geq 4 k+5$. Let $Z \subset X$ be a zero-dimensional subscheme of length $k+1$. Then either $K_{X}+N$ is $k$-very ample at $Z$ or there exists an effective divisor $D$ containing $Z$ such that $N-2 D$ is $\mathbb{Q}$-effective (i.e. there exists a positive integer $m$ such that $|m(N-2 D)| \neq \emptyset$ ) and the following inequalities are satisfied:

$$
N D-k-1 \leq D^{2}<\frac{N D}{2}<k+1
$$

\section{Nagata Conjecture and Seshadri Constants}

The following conjecture was formulated by Nagata [18] over forty years ago.

Nagata Conjecture. Let $P_{1}, \ldots, P_{r}$ be $r \geq 9$ general points in $\mathbb{P}^{2}$ and let $k_{1}, \ldots, k_{r}$ be fixed non-negative integers. If $C \subset \mathbb{P}^{2}$ is a curve of degree $d$ such that mult $_{P_{i}} C \geq$ $k_{i}$, then

$$
d \geq \frac{1}{\sqrt{r}} \sum_{i=1}^{r} k_{i}
$$


It still remains open, apart from the case when the number of blown up points $r$ is a square, which was settled by Nagata himself. In fact if $r$ is a square and $r \geq 16$ Nagata proved that the sharp inequality holds for very general points $P_{1}, \ldots, P_{r}$ (which was essential for his counterexample to the Hilbert's 14th problem). In the remaining cases (i.e. $r$ not a square) both formulations are of course equivalent. For the purpose of this paper it is convenient to formulate the Nagata Conjecture in the language of Seshadri constants.

We recall briefly that Seshadri constants were introduced by Demailly [10 in order to measure the local positivity of a line bundle.

Definition 2.1. Let $X$ be a smooth projective variety and $P_{1}, \ldots, P_{r}$ pairwise distinct points in $X$. The $r$-tuple Seshadri constant of $L$ at $P_{1}, \ldots, P_{r}$ is the number

$$
\varepsilon\left(L ; P_{1}, \ldots, P_{r}\right)=\sup \left\{\varepsilon \text { such that } \pi^{*} L-\varepsilon \sum_{i=1}^{r} E_{i} \text { is nef }\right\},
$$

where $\pi$ is the blowing up of $X$ at $P_{1}, \ldots, P_{r}$ with exceptional divisors $E_{1}, \ldots, E_{r}$.

The Nagata Conjecture now states (see 23], 2])

Nagata Conjecture, second formulation. Let $P_{1}, \ldots, P_{r}$ be $r \geq 9$ very general points in $\mathbb{P}^{2}$. Then

$$
\varepsilon\left(\mathcal{O}_{\mathbb{P}^{2}}(1) ; P_{1}, \ldots, P_{r}\right)=\frac{1}{\sqrt{r}} .
$$

Note that the inequality $\varepsilon\left(\mathcal{O}_{\mathbb{P}^{2}}(1) ; P_{1}, \ldots, P_{r}\right) \leq \frac{1}{\sqrt{r}}$ is obvious, so only the other inequality is of interest. It might be also worthwhile here to observe that in the above formulation the case when the number of points $r$ is a square $r=s^{2}$ is easy to prove. Indeed, let $C$ be a smooth curve of degree $s$ and let $P_{1}, \ldots, P_{s^{2}}$ be arbitrary points on $C$. Since $C$ is in particular irreducible we check that the Seshadri constant $\varepsilon\left(\mathcal{O}_{\mathbb{P}^{2}}(1) ; P_{1}, \ldots, P_{s^{2}}\right)=\frac{1}{s}$. In fact if it were smaller, say equal $\varepsilon<\frac{1}{s}$, then there would be a curve $D$ passing through $P_{1}, \ldots, P_{s^{2}}$ such that its proper transform on the blow-up would spoil the nefness of $H-\frac{1}{s} \sum_{i=1}^{s^{2}} E_{i}$. But on $\mathbb{P}^{2}$ this would imply that $D$ has a negative intersection with $C$, which is absurd. This shows that the Seshadri constant is maximal for the special choice of points $P_{1}, \ldots P_{s^{2}}$ and then by the semi-continuity result in the spirit of [19] we conclude that it must be maximal for a very general set of $s^{2}$ points.

It seems that when $r$ is not a square the best general bound known up to now is the following result of $\mathrm{Xu}$ [21, Theorem 1(a)].

Proposition 2.2 (Xu). Let $P_{1}, \ldots, P_{r}$ be $r \geq 9$ general points in $\mathbb{P}^{2}$. Then

$$
\varepsilon\left(\mathcal{O}_{\mathbb{P}^{2}}(1) ; P_{1}, \ldots, P_{r}\right) \geq \frac{\sqrt{r-1}}{r} .
$$

It should be pointed out that for special values of $r$ there are various results e.g. 6], 13], [14] which imply better bounds (also often better than those of Theorem 2). However such results lack the uniformity of the above Proposition and of Theorem 2. Xu's approach is based on the following observation (which appears implicitly already in [12]) which is crucial also for our consideration.

Lemma $2.3(\mathrm{Xu})$. Let $P_{1}, \ldots, P_{s}$ be general points in $\mathbb{P}^{2}$ and let $C$ be a reduced and irreducible curve of degree $p$ passing through the points $P_{i}$ with the multiplicity 
$\operatorname{mult}_{P_{i}} C=m_{i}$, for $i=1, \ldots, s$. Then

$$
p^{2} \geq \sum_{i=1}^{s} m_{i}^{2}-m_{j}
$$

for arbitrary $j \in\{1, \ldots, s\}$ with $m_{j}>0$.

\section{BlOW-UPS OF $\mathbb{P}^{2}$}

We are now in a position to formulate and prove the main results of this paper.

Theorem 1. Let $\pi: X \longrightarrow \mathbb{P}^{2}$ be the blowing up of $\mathbb{P}^{2}$ in $r$ general points and let $k$ and $d$ be non-negative integers such that $d \geq 4 k+1$. If $r \leq \frac{(d+3)^{2}}{(k+1)^{2}}-4$, then the line bundle $L=d H-k \sum_{i=1}^{r} E_{i}$ is $k$-very ample.

Proof. The idea is to look at $L$ as an adjoint line bundle and with help of inequalities from Proposition 1.3 show that no curve $D$ spoiling the $k$-very ampleness of $L$ can exist. Since for $k=0$ and $k=1$ the Theorem is known we assume that $k \geq 2$.

Turning to the details, let $N=L-K_{X}=(d+3) H-(k+1) \sum_{i=1}^{r} E_{i}$. First we show that $N$ satisfies assumptions of Proposition 1.3. First of all we have

$$
N^{2}=(d+3)^{2}-r(k+1)^{2} \geq 4(k+1)^{2}>4 k+5 .
$$

Our next claim is that

$N . C \geq k+1$ for every irreducible and reduced curve $C \subset X$ and the equality holds only if $C$ is one of the exceptional divisors $E_{i}$.

Taking this for granted for a while, the theorem follows immediately. Indeed, $N$ is then obviously nef so that Proposition 1.3 applies. Assuming that $L=K_{X}+N$ fails to be $k$-very ample we are given a curve $D$ in $X$ satisfying

$$
N . D-k-1 \leq D^{2}<\frac{N . D}{2}<k+1 \text {. }
$$

Now (3.1), together with the first inequality, implies that $D^{2} \geq 0$ and $D^{2}=0$ if and only if $D$ consists of the exceptional divisors. Since these are disjoint this implies in turn $D^{2}<0$, a contradiction. So we are left with $D^{2} \geq 1$ but then the Hodge Index Theorem gives

$$
1 \cdot\left(4(k+1)^{2}\right) \leq D^{2} \cdot N^{2} \leq(N . D)^{2} .
$$

Hence $N . D \geq 2(k+1)$ which contradicts the last of the above inequalities.

It remains to show (3.1). If $C$ is one of the exceptional divisors the claim is obvious, so we may assume that $C$ is of the form $C=\pi^{*} F-\sum_{i=1}^{r} m_{i} E_{i}$, where $F$ is a reduced and irreducible plane curve of degree $p$. Renumbering the points if necessary we may assume that $m_{1} \geq \cdots \geq m_{s} \geq 1$ and $m_{s+1}=\cdots=m_{r}=0$. Then

$$
N . C=(d+3) p-(k+1) \sum_{i=1}^{s} m_{i}
$$

and we claim that

$$
(d+3) p>(k+1)\left(\sum_{i=1}^{s} m_{i}+1\right)
$$


Since by our assumption $\frac{d+3}{k+1} \geq \sqrt{r+4} \geq \sqrt{s+4}$ it suffices to show that

$$
p \sqrt{s+4}>\sum_{i=1}^{s} m_{i}+1
$$

Now we have to consider several cases.

Case 1a. We assume that $m_{s}=1$ and $s \geq 6$. Under this assumption (3.3) is equivalent to

$$
p \sqrt{s+4}>\sum_{i=1}^{s-1} m_{i}+2 .
$$

Since $\left(\sum_{i=1}^{s-1} m_{i}\right)^{2} \leq(s-1) \sum_{i=1}^{s-1} m_{i}^{2}$ it is enough to show

$$
(s-1) \sum_{i=1}^{s-1} m_{i}^{2}<p^{2}(s+4)+4-4 p \sqrt{s+4} .
$$

From Lemma 2.3 we have $\sum_{i=1}^{s-1} m_{i}^{2}+1 \leq p^{2}+1$, hence our inequality follows from

$$
5 p^{2}-4 p \sqrt{s+4}+4>0
$$

which is easily seen to hold for $p \geq \sqrt{s-1}$ (Lemma 2.3 again) and $s \geq 6$.

Case 1b. We assume that $m_{s}=1$ and $s \leq 5$. Using our assumption $d \geq 4 k+1$ and (3.2) it is enough to show

$$
4 p>\sum_{i=1}^{s} m_{i}+1
$$

Since $C$ is irreducible either $s \leq 2$ and $m_{1}=m_{s}=1$ in which case we are done, or $p \geq m_{1}+1$. But then we have

$$
4 p \geq 4 m_{1}+4 \geq \sum_{i=1}^{s-1} m_{i}+4>\sum_{i=1}^{s-1} m_{i}+2=\sum_{i=1}^{s} m_{i}+1
$$

and this shows (3.4).

Case 2a. We assume that $m_{s} \geq 2$ and $s \geq 4$. Since $\left(\sum_{i=1}^{s} m_{i}\right)^{2} \leq s \sum_{i+1}^{s} m_{i}^{2}$ we see from (3.3) that it is enough to prove

$$
s \sum_{i=1}^{s} m_{i}^{2}<p^{2}(s+4)+1-2 p \sqrt{s+4} .
$$

From Lemma 2.3 we have $\sum_{i=1}^{s} m_{i}^{2} \leq p^{2}+m_{s}$ hence we are reduced to showing that

$$
s m_{s}+2 p \sqrt{s+4}<4 p^{2}+1 .
$$

Now looking at Lemma 2.3 in a slightly different way, we have

$$
p^{2} \geq \sum_{i=1}^{s} m_{i}^{2}-m_{s} \geq(s-1) m_{s}
$$

or equivalently $s m_{s} \leq s \frac{p^{2}}{s-1}$. Hence it is enough to show

$$
2 p \sqrt{s+4}<\left(4-\frac{s}{s-1}\right) p^{2}+1 .
$$


Since (3.5) implies in particular that $p \geq \sqrt{2(s-1)}$ the inequality in question reduces finally to

$$
\sqrt{2(s+4)}<\frac{3 s-4}{\sqrt{s-1}}
$$

which is easy to check for $s \geq 4$.

Case 2b. Finally we assume that $m_{s} \geq 2$ and $s \leq 3$. This is easy and goes e.g. as Case 1b.

As already mentioned in the Introduction we expect that the above Theorem is not optimal. A natural generalization of results of Coppens and D'Almeida and Hirschowitz would be the following.

Conjecture. Let $\pi: X \longrightarrow \mathbb{P}^{2}$ be the blowing up of $\mathbb{P}^{2}$ in $r$ general points and let $k$ and $d$ be non-negative integers such that $d \geq 4 k+1$. If $r$ satisfies

$$
\left(\begin{array}{c}
d+2 \\
2
\end{array}\right)-r\left(\begin{array}{c}
k+1 \\
2
\end{array}\right) \geq 3(k+1)
$$

then the line bundle $L=d H-k \sum_{i=1}^{r} E_{i}$ is $k$-very ample.

This means that given a fixed $k$ for $d$ sufficiently large the line bundle $L$ is $k$ very ample as soon as it has enough sections (the dimension of the $(k+1)$-st secant variety of $X$ must be the expected one under a $k$-very ample embedding). The referee informed us that Alexander had a similar conjecture but we were not able to track it down in the literature.

Remark 3.1. The above Conjecture implies the Nagata Conjecture (for $r \geq 17$ ).

Indeed, let $r \geq 17$ be fixed. Then given $k$ we compute the minimal $d_{k}$ satisfying (3.6) and $d \geq 4 k+1$ (the bound $r \geq 17$ is in fact caused by the latter condition). We have $d_{k}=\left\lceil\frac{-3+\sqrt{1+4(6+r k)(k+1)}}{2}\right\rceil$. Of course a $k$-very ample line bundle is nef, so $\varepsilon\left(\mathcal{O}_{\mathbb{P}^{2}}(1) ; P_{1}, \ldots, P_{r}\right) \geq \frac{k}{d_{k}}$ for every $k$. Passing with $k$ to infinity we get $\varepsilon\left(\mathcal{O}_{\mathbb{P}^{2}}(1) ; P_{1}, \ldots, P_{r}\right) \geq \frac{1}{\sqrt{r}}$.

Using similar methods as in the proof of Theorem 1 we show a criterion for the ampleness of a line bundle of the form $L=d H-k \sum_{i=1}^{r} E_{i}$ which in turn is used in the proof of Theorem 2.

Theorem 3. Let $\pi: X \longrightarrow \mathbb{P}^{2}$ be the blowing up of $\mathbb{P}^{2}$ in $r$ general points and let $k \geq 2$ and $d$ be integers such that $d \geq 3 k+1$. If $r \leq \frac{d^{2}}{k^{2}}-1$, then the line bundle $L=d H-k \sum_{i=1}^{r} E_{i}$ is ample.

Proof. The idea is to apply the Nakai-Moishezon criterion. First of all we have

$$
N^{2}=d^{2}-r k^{2} \geq k^{2}>0 .
$$

Next we claim that N.C>0 for all irreducible and reduced curves $C \subset X$. This is obvious if $C$ is one of the exceptional curves so we may assume that $C=\pi^{*} F-\sum_{i=1}^{r} m_{i} E_{i}$ where $F$ is a reduced and irreducible plane curve of degree $p$. Furthermore without loss of generality we may assume that $m_{1} \geq \cdots \geq m_{s} \geq 1$ and $m_{s+1}=\cdots=m_{r}=0$. Computing $N$.C we see that our claim is equivalent to

$$
d p>k \sum_{i=1}^{s} m_{i}
$$


Since $\frac{d}{k} \geq \sqrt{r+1} \geq \sqrt{s+1}$ and $\left(\sum_{i=1}^{s} m_{i}\right)^{2} \leq s \sum_{i=1}^{s} m_{i}^{2}$ it suffices to show that

$$
s \sum_{i=1}^{s} m_{i}^{2}<p^{2}(s+1) .
$$

From Lemma 2.3 we have

$$
\sum_{i=1}^{s} m_{i}^{2} \leq p^{2}+m_{s}
$$

so that we are done if

$$
s m_{s}<p^{2}
$$

holds. In fact (3.8) implies (3.9) unless $m_{1}=\cdots=m_{s}=1$. In the latter case in (3.7) we have to show

$$
p \frac{d}{k}>s
$$

If $s \leq 5$, then using the assumption $d \geq 3 k+1$ it is enough to show $3 p \geq s$, which follows by the assumption that the points $P_{1}, \ldots, P_{r}$ are general. So we assume that $s \geq 6$. Since $\frac{d}{k} \geq \sqrt{s+1}$ it is enough to prove that

$$
p>\frac{s}{\sqrt{s+1}} .
$$

From the generality assumption again we have $\left(\begin{array}{c}p+2 \\ 2\end{array}\right)-s \geq 1$ or equivalently $p \geq$ $\frac{\sqrt{8 s+9}-3}{2}$. Now the claim follows from the simple observation that the real valued function

$$
f(s)=\frac{\sqrt{8 s+9}-3}{2}-\frac{s}{\sqrt{s+1}}
$$

is positive for $s \geq 6$.

Now we pass to the proof of our global generation result. In the case of fat points, i.e. $k \geq 2$, this result seems to be new. Moreover it implies Theorem 2 . As pointed out by the referee the global generation problem is connected to the vanishing of the cohomology groups of line boundles of the type considered here (see [15] for a conjectural picture concerning vanishing of $H^{1}$ and 17] for partial results). As the vanishing follows from the positivity for many special values of $r$ one can get better bounds than those presented below using certain results of [6], [13, [14.

Theorem 4. Let $\pi: X \longrightarrow \mathbb{P}^{2}$ be the blowing up of $\mathbb{P}^{2}$ in $r$ general points and let $k \geq 2$ and $d$ be integers such that $d \geq 3 k+1$. If $r \leq \frac{(d+3)^{2}}{(k+1)^{2}}-1$, then the line bundle $L=d H-k \sum_{i=1}^{r} E_{i}$ is globally generated.

Proof. As in the proof of Theorem 1 let $N=L-K_{X}=(d+3) H-(k+1) \sum_{i=1}^{r} E_{i}$. Then Theorem 3 implies that $N$ is ample and satisfies $N^{2}>5$ so that Proposition 1.3 applies. This means that if $L=K_{X}+N$ fails to be globally generated, then there exists a curve $D \subset X$ such that $N . D=1$ and $D^{2}=0$. Let $p, m_{1}, \ldots, m_{r}$ 
be integers such that $D \equiv p H-\sum_{i=1}^{r} m_{i} E_{i}$. Computing $N . D$ we get $(d+3) p=$ $(k+1) \sum_{i=1}^{r} m_{i}+1$ which implies $\sum_{i=1}^{r} m_{i}>0$, so that in fact it must be

$$
\sum_{i=1}^{r} m_{i} \geq 1 \text {. }
$$

Taking into account $\frac{d+3}{k+1} \geq \sqrt{r+1}$ we have

$$
p \sqrt{r+1} \leq \sum_{i=1}^{r} m_{i}+\frac{1}{k+1} .
$$

Since $D^{2}=0$ we have $p^{2}=\sum_{i=1}^{r} m_{i}^{2}$, hence

$$
(r+1) \sum_{i=1}^{r} m_{i}^{2} \leq\left(\sum_{i=1}^{r} m_{i}+\frac{1}{k+1}\right)^{2} .
$$

Now, the right-hand side is bounded by $r \sum_{i=1}^{r} m_{i}^{2}+\frac{2}{k+1} \sum_{i=1}^{r} m_{i}+\frac{1}{(k+1)^{2}}$ so that

$$
(k+1)^{2} \sum_{i=1}^{r} m_{i}^{2} \leq 2(k+1) \sum_{i=1}^{r} m_{i}+1 .
$$

Since $\sum_{i=1}^{r} m_{i}^{2} \geq \sum_{i=1}^{r} m_{i}$ we get $\left(k^{2}-1\right) \sum_{i=1}^{r} m_{i} \leq 1$ which in view of $k \geq 2$ and (3.10) gives a contradiction.

Finally as an easy corollary we obtain

Theorem 2. Let $P_{1}, \ldots, P_{r}$ be $r \geq 9$ general points in $\mathbb{P}^{2}$ and let $k_{1}, \ldots, k_{r}$ be fixed non-negative integers. If $C \subset \mathbb{P}^{2}$ is a curve of degree $d$ such that mult $_{P_{i}} C \geq k_{i}$, then

$$
d \geq \frac{1}{\sqrt{r+1}} \sum_{i=1}^{r} k_{i}
$$

Proof. This goes exactly as in Remark 3.1 Using Theorem 3 we have $d_{k}=$ $\lceil(k+1) \sqrt{r+1}\rceil-3$ this time.

Remark 3.2. After this paper was written we learned that Harbourne 14, Proposition I.2] obtained the same bound in a much broader context of all polarized surfaces of any characteristic.

\section{ACKNOWLEDGMENTS}

This paper was written while the first author was a Fulbright Fellow at the University of Michigan. It is a pleasure to thank Rob Lazarsfeld for his hospitality and interesting discussions and the Fulbright Foundation for its financial support.

The second author thanks Hélène Esnault and Eckart Viehweg for creating a very friendly atmosphere while she visited Essen and for many discussions.

We would also like to thank Brian Harbourne for interesting e-mail exchange and the referee for the very careful reading of the manuscript and remarks which allowed us to improve the exposition and put the results in a better perspective. 


\section{REFERENCES}

[1] Ballico, E., Coppens, M., Very ample line bundles on blown-up projective varieties. Bull. Belg. Math. Soc. 4 (1997), 437-447. MR 98k:14019

[2] Bauer, Th., Seshadri constants on algebraic surfaces. Math. Ann. 313, (1999), 547-583. MR 2000d:14006

[3] Beltrametti, M.C., Sommese, A.J.: Zero cycles and $k$-th order embeddings. Projective surfaces and their classification, Symp. Math., INDAM 32, Academic Press, 1988, pp 33-48. MR 95d:14005

[4] Bese, E., On the spannedness and very ampleness of certain line bundles on the blow-ups of $\mathbb{P}_{\mathbb{C}}^{2}$ and $\mathbb{F}_{r}$. Math. Ann. 262 (1983), 225-238. MR 84g:14006

[5] Biancofiore, A., On the hyperplane sections of blow-ups of complex projective plane. Can. J. Math. 41 (1989), 1005-1020. MR 90m:14007

[6] Biran, P., Constructing new ample divisors out of old ones. Duke Math. J. 98 (1999), 113-135. MR 2000d:14047

[7] Catanese, F., Göttsche, L.: d-very ample line bundles and embeddings of Hilbert schemes of o-cycles. Manuscripta Mat. 68 (1990) 337-341. MR 91i:14012

[8] Coppens, M., Embeddings of general blowing-ups at points. J. Reine Angew. Math. 469 (1995), 179-198. MR 97d:14008

[9] D'Almeida, J., Hirschowitz, A.: Quelques plongements projectifs non speciaux de surfaces rationelles. Math. Z. 211 (1992), 479-483. MR 94a:14012

[10] Demailly, J.-P., Singular Hermitian metrics on positive line bundles. Lect. Notes Math. 1507, Springer, 1992, pp. 87-104. MR 93g:32044

[11] Di Rocco, S.: k-very ample line bundles on Del Pezzo surfaces. Math. Nachr. 179 (1996), 47-56. MR 97h:14007

[12] Ein, L., Lazarsfeld, R.: Seshadri constants on smooth surfaces. Journes de Geometrie Algebrique d'Orsay (Orsay, 1992). Asterisque No. 218 (1993), 177-186. MR 95f:14031

[13] Harbourne, B.: On Nagata's Conjecture. J. Alg. 236 (2001), 692-702. MR 2002b:14039

[14] Seshadri constants and very ample divisors on algebraic surfaces. Preprint alg-geom 0103029.

[15] Hirschowitz, A.: Une conjecture pour la cohomologie des diviseurs sur le surfaces rationelles génériques. J. Reine Angew. Math. 397 (1989), 208-213. MR 90g:14021

[16] Küchle, O.: Ample line bundles on blown up surfaces. Math. Ann. 304 (1996), 151-155. MR 97a:14041

[17] Mignon, Th.: Systèmes de courbes planes à singularités imposées: le cas des multiplicités inférieures ou égales à quatre. J. Pure Appl. Algebra 151 (2000), 173-195. MR 2001g:14048

[18] Nagata, M., On the 14-th problem of Hilbert. Am. J. Math. 81 (1959), 766-772. MR 21:4151

[19] Oguiso, K., Seshadri constant for a family of surfaces. Preprint alg-geom 0002211.

[20] Tutaj-Gasińska, H.: Embeddings of blown-up plane. Geom. Dedicata 76 (1999), 165-175. MR 2000h:14006

[21] Xu, G., Curves in $\mathbb{P}^{2}$ and symplectic packings. Math. Ann. 299 (1994), 609-613. MR 95f: 14058

[22] , Divisors on the blow-up of the projective plane. Manuscripta Math. 86 (1995), 195197. MR 96c:14005

[23] _ Ample line bundles on smooth surfaces. J. Reine Angew. Math. 469 (1995), 199-209. MR 96k:14003

Instytut Matematyki, Uniwersytet Jagielloński, Reymonta 4, PL-30-059 Kraków, PolAND

Current address: Universität GH Essen, FB 6 Mathematik, D-45117 Essen, Germany

E-mail address: szemberg@im.uj.edu.pl

Instytut Matematyki, Uniwersytet Jagielloński, Reymonta 4, PL-30-059 Kraków, POLAND

E-mail address: htutaj@im.uj.edu.pl 